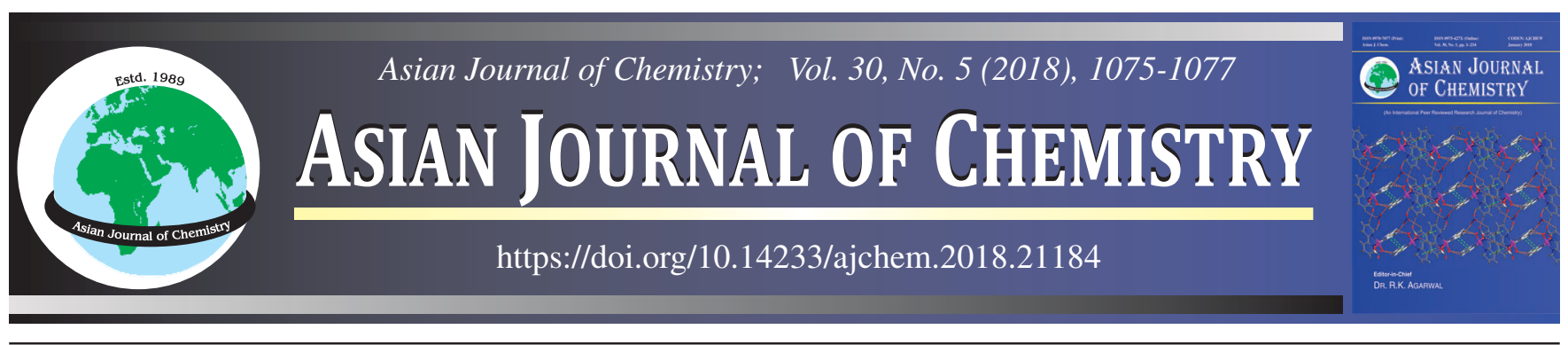

\title{
Reactions of Huisgen Zwitterions with Diphenyl Cyclopropenone: A Novel Strategy for the Synthesis of Oxazinone Derivatives
}

\section{R. Divya Mohan ${ }^{1, *}$ and AnU Jose ${ }^{2}$}

${ }^{1}$ Department of Chemistry, Amrita School of Arts and Sciences, Amrita Vishwa Vidyapeetham, Amritapuri-690 525, India

${ }^{2}$ Department of Chemistry, Providence Women's College, Calicut-673 009, India

*Corresponding author: E-mail: divyamohanr@hotmail.com

Received: 15 December 2017; Accepted: 15 February 2018; Published online: 29 March 2018;

A facile and novel route for the synthesis of diphenyl oxazinones in good yield was developed using diphenyl cyclopropenone and dialkyl azodicarboxylate with triphenyl phosphine as catalyst at room temperature and are well characterized using spectroscopic studies.

Keywords: Oxazinones, Diphenyl cyclopropenone, Dialkyl azodicarboxylate.

\section{INTRODUCTION}

In synthetic organic chemistry carbon hetero-atom and carbon carbon bond forming reactions are of prior importance. Generally polar and pericyclic reaction strategies are used for this which utilize reactive intermediates like carbanions, enols, radicals, carbenes, zwitterions, etc. Although potentially very useful, zwitterions have received less attention from this perspective. The present work is concerned with the use of a less well-known reactive intermediate viz., zwitterion. Neutral nucleophiles like triphenyl phosphine, nucleophilic carbenes, and isocyanides can form zwitterionic intermediates with azodicarboxylates and activated acetylenes [1-4].

Although, phosphine-azoester zwitterion generally known as the Huisgen zwitterion [5], has been known in the literature for almost five decades, barring its use as nucleophilic trigger in the Mitsunobu reaction [6-8]. The chemistry of these powerful reactive intermediates remained largely unexplored. In recent years, our research group has explored the synthetic potential of these zwiterionic intermediates with a view to synthesize a variety of heterocycles $[1,2]$ and uncovered the interesting reactivity patterns of the zwitterions generated from triphenyl phosphine and dialkyl azodicarboxylate. In continuation these studies, presently, we investigated the reactions of Huisgen zwitterions derived from triphenylphosphine-azodicarboxylate with diphenyl cyclopropenones leading to the formation of oxazinones. Cyclopropenones are an important class of compounds because of their application in a wide range of reactions such as decarbonylation, addition, oxidation, substitution reactions, etc. Further, the variety of reactions for such a simple system as cyclopropenone has also led to the incorporation of phosphine azoester into the cyclopropenone system.

\section{EXPERIMENTAL}

${ }^{1} \mathrm{H}$ NMR spectra are recorded at 300 and $500 \mathrm{MHz}$, respectively and ${ }^{13} \mathrm{C}$ spectrum at $125 \mathrm{MHz}$ using Bruker Avance DPX$500 \mathrm{MHz}$ NMR Spectrometer. Chemical shift values $(\delta)$ are reported with respect to TMS $\left({ }^{1} \mathrm{H}\right)$ and $\mathrm{CDCl}_{3}\left({ }^{12} \mathrm{C}\right)$ as internal standards while coupling constant values $(J)$ are reported in hertz $(\mathrm{Hz})$. IR spectra are recorded with Bomem MB Series FTIR spectrophotometer. Mass spectra are recorded with FAB/ LRMS and EI/HRMS using JEOL mass spectrometer. Diethyl azodicarboxylate, diisopropyl azodicaboxylate and dibenzyl azodicarboxylate are purchased from Lancaster Chemical Co. and are used as such. Triphenylphosphine is purchased from Merck. Organic solvents are distilled before use. Thin layer chromatography is done using glass plates with silica gel coating having calcium sulfate as binder material. Column chromatography is performed with silica gel (100-200 mesh) using hexaneethyl acetate mixture for elution.

Diphenyl cyclopropenone was prepared by employing known procedures [9]. 3,3'-Dinitrodiphenyl cyclopropenone and 3,3'dibromodiphenyl cyclopropenone were obtained from diphenylcyclopropenone by aromatic electrophilic substitution reactions [10,11]. 4,4'-Dichlorodiphenyl cyclopropenones are prepared starting from their corresponding para substituted phenyl acetic acids [12].

General procedure for the synthesis of 2-alkoxy-4,5-diaryl6H-1,3-oxazin-6-one: These were obtained from the reaction of the corresponding diaryl cyclopropenone $(0.25 \mathrm{mmol})$ with 
dialkyl azodicaboxylate $(4 \mathrm{mmol})$ in dichloromethane (DCM) in the presence of triphenylphosphine ( $4 \mathrm{mmol})$ at room temperature for 15 min under argon atmosphere (Scheme-I). The product was isolated from the mixture with the help of column chromatography using hexane and ethyl acetate (95:5) as eluent. The reaction was found general for a number of diphenyl cyclpropenones prepared from dibenzyl ketones as shown in Table-1.

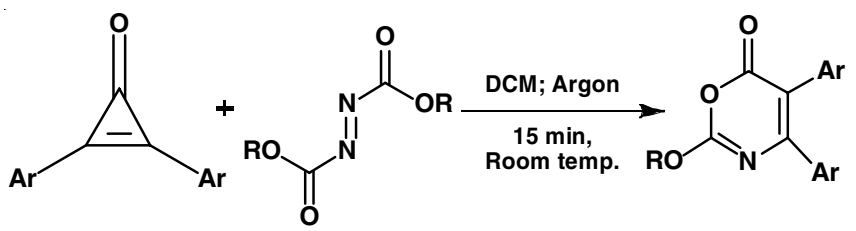

Scheme-I

\begin{tabular}{cllc}
\multicolumn{5}{c}{ TABLE-1 } \\
\multicolumn{5}{c}{$\begin{array}{c}\text { REACTIONS OF DIARYL CYCLOPROPENONES } \\
\text { WITH DIALKYL AZODICARBOXYLATES }\end{array}$} \\
\hline Compound & \multicolumn{1}{c}{ Ar group } & \multicolumn{1}{c}{ R group } & Yield (\%) \\
\hline 1 & Phenyl & Isopropyl & 63 \\
2 & Phenyl & Ethyl & 68 \\
3 & Phenyl & Benzyl & 65 \\
4 & $m$-Nitro phenyl & Isopropyl & 56 \\
5 & $m$-Nitro phenyl & Ethyl & 65 \\
6 & $p$-Chloro phenyl & Isopropyl & 85 \\
7 & $p$-Chloro phenyl & Ethyl & 75 \\
8 & $p$-Chloro phenyl & Benzyl & 50 \\
9 & $m$-Bromo phenyl & Ethyl & 75 \\
10 & $m$-Bromo phenyl & Isopropyl & 70 \\
\hline
\end{tabular}

\section{Spectral data}

2-Isopropoxy-4,5-diphenyl-6H-1,3-oxazin-6-one (1): White solid; IR (KBr, $\left.v_{\max }, \mathrm{cm}^{-1}\right): 1746,1607,1096$ and 1295; ${ }^{1} \mathrm{H}$ NMR $\left(500 \mathrm{MHz} \mathrm{CDCl}_{3}\right): \delta$ 7.17-7.27 (m, 10H), 5.37-5.39 $(\mathrm{m}, 1 \mathrm{H}, J=6 \mathrm{~Hz}), 1.47-1.48(\mathrm{~d}, 6 \mathrm{H}, J=6.5 \mathrm{~Hz}) ;{ }^{13} \mathrm{CNMR}(75.47$ $\left.\mathrm{MHz}, \mathrm{CDCl}_{3}\right): \delta 161.2,160.3,157.1,136.5,133.0,130.8,128.3$, 127.8, 113, 74.7, $21.6 \mathrm{ppm}$. LRMS (+FAB) $\mathrm{m} / \mathrm{z}$ calcd for $\mathrm{C}_{19} \mathrm{H}_{17} \mathrm{NO}_{3}(\mathrm{M}+\mathrm{H})^{+}:$308.12; Found: 308.58 .

2-Ethoxy-4,5-diphenyl-6H-1,3-oxazin-6-one (2): White solid; IR (KBr, $\left.v_{\max }, \mathrm{cm}^{-1}\right)$ : 1754, 1614, 1319 and 1096; ${ }^{1} \mathrm{H}$ NMR (500 MHz CDCl $)_{3}$ : $\delta 7.18-7.33(\mathrm{~m}, 10 \mathrm{H}), 4.56-4.57(\mathrm{q}$, $2 \mathrm{H}, J=7 \mathrm{~Hz}), 1.47-1.5(\mathrm{t}, 3 \mathrm{H}, J=6.5 \mathrm{~Hz}) ;{ }^{13} \mathrm{C}$ NMR $(75.47$ $\left.\mathrm{MHz}, \mathrm{CDCl}_{3}\right): \delta 161.11,160.1,157.5,136.4,132.9,130.8,130$, 128.3, 127.9, 127.8, 66.3, $14.1 \mathrm{ppm}$. LRMS (+FAB) $\mathrm{m} / 2$ calcd for $\mathrm{C}_{18} \mathrm{H}_{15} \mathrm{NO}_{3}(\mathrm{M}+\mathrm{H})^{+}$: 294.11; Found: 294.50.

2-(Benzyloxy)-4,5-diphenyl-6H-1,3-oxazin-6-one (3): White solid; IR $\left(\mathrm{KBr}, v_{\max }, \mathrm{cm}^{-1}\right): 1752,1612,1305$ and 1115; ${ }^{1} \mathrm{H} \mathrm{NMR}\left(500 \mathrm{MHz}, \mathrm{CDCl}_{3}\right): \delta 7.17-7.47(\mathrm{~m}, 15 \mathrm{H}), 5.51(\mathrm{~s}, 2 \mathrm{H})$; ${ }^{13} \mathrm{C}$ NMR (75.47 MHz, $\left.\mathrm{CDCl}_{3}\right): \delta 160.9,160.0,157.5,136.3$, $134.3,132.8,130.8,130,129.9,129,127.9,113.5,71.5 \mathrm{ppm}$. LRMS (+FAB) $m / z$ calcd for $\mathrm{C}_{23} \mathrm{H}_{17} \mathrm{NO}_{3}(\mathrm{M}+\mathrm{H})^{+}: 356.12$; Found: 356.67 .

2-Isopropoxy-4,5-bis(4-chlorophenyl)- $6 \boldsymbol{H}$-1,3-oxazin6-one (4): White solid; IR $\left(\mathrm{KBr}, v_{\max }, \mathrm{cm}^{-1}\right)$ : 1759, 1608, 1091 and $1309 ;{ }^{1} \mathrm{HNMR}\left(300 \mathrm{MHz}, \mathrm{CDCl}_{3}\right): \delta$ 7.91-7.11 (m, 8H), 5.4$5.25(\mathrm{~m}, 1 \mathrm{H}, J=6.3 \mathrm{~Hz}), 1.46-1.450(\mathrm{~d}, 6 \mathrm{H}, J=6.3 \mathrm{~Hz}) ;{ }^{13} \mathrm{CNMR}$ (125 MHz, $\left.\mathrm{CDCl}_{3}\right): \delta 160.3,159.8,157.3,136.4,134.6,134.1$, $1.32 .1,128.9,128.3,112,21.59 \mathrm{ppm}$. LRMS (+FAB) $\mathrm{m} / \mathrm{z}$ calcd for $\mathrm{C}_{19} \mathrm{H}_{15} \mathrm{NO}_{3} \mathrm{Cl}_{2}(\mathrm{M}+\mathrm{H})^{+}$: 376.04; Found: 376.34 .
2-Ethoxy-4,5-bis(4-chlorophenyl)-6H-1,3-oxazin-6-one (5): White solid; IR (KBr, $\left.v_{\max }, \mathrm{cm}^{-1}\right)$ : 1753, 1611, 1311 and 1091; ${ }^{1} \mathrm{H}$ NMR (500 MHz, $\left.\mathrm{CDCl}_{3}\right): \delta 7.11-7.31(\mathrm{~m}, 8 \mathrm{H}), 4.53-$ 4.60 (q, $2 \mathrm{H}, J=7.5 \mathrm{~Hz}), 1.47-1.5(\mathrm{t}, 3 \mathrm{H}, J=7.5 \mathrm{~Hz}) ;{ }^{13} \mathrm{C} \mathrm{NMR}$ $\left(75.47 \mathrm{MHz}, \mathrm{CDCl}_{3}\right): \delta 159.7,157.7,136.5,134.5,134.2,132.1$, 131.2, 128.9, 128.4, 112.3, 66.6, 14.1 ppm. LRMS (+FAB) $\mathrm{m} / \mathrm{z}$ calcd for $\mathrm{C}_{18} \mathrm{H}_{13} \mathrm{NO}_{3} \mathrm{Cl}_{2}(\mathrm{M}+\mathrm{H})^{+}$: 362.03; Found: 362.38 .

2-(Benzyloxy)-4,5-bis(4-chlorophenyl)-6H-1,3-oxazin6-one (6): White solid; IR ( $\left.\mathrm{KBr}, v_{\max }, \mathrm{cm}^{-1}\right)$ : 1741 and 1611; ${ }^{1} \mathrm{H} \mathrm{NMR}\left(500 \mathrm{MHz}, \mathrm{CDCl}_{3}\right): \delta 7.41-7.10(\mathrm{~m}, 13 \mathrm{H}), 5.50(\mathrm{~s}, 2 \mathrm{H})$; ${ }^{13} \mathrm{C}$ NMR $\left(75.47 \mathrm{MHz}, \mathrm{CDCl}_{3}\right): \delta 161.0,160.1,157.4,136.3$, $134.3,132.8,130.8,130,129.9,129,128.5,127.9,113.5,71.5$ ppm. LRMS (+FAB) $m / z$ calcd for $\mathrm{C}_{23} \mathrm{H}_{15} \mathrm{NO}_{3} \mathrm{Cl}_{2}(\mathrm{M}+\mathrm{H})^{+}: 425.28$; Found: 425.69 .

2-Isopropoxy-4,5-bis(3-nitrophenyl)- 6H-1,3-oxazin-6one (7): White solid; IR $\left(\mathrm{KBr}, v_{\max }, \mathrm{cm}^{-1}\right): 1759,1609,1096$ and $1295 ;{ }^{1} \mathrm{H}$ NMR $\left(300 \mathrm{MHz}, \mathrm{CDCl}_{3}\right)$ : $\delta 7.8-7.2(\mathrm{~m}, 10 \mathrm{H})$, 5.18-5.14 (m, $1 \mathrm{H}), 1.35(\mathrm{~d}, 6 \mathrm{H}, J=6.5 \mathrm{~Hz}) ;{ }^{13} \mathrm{C} \mathrm{NMR}(125 \mathrm{MHz}$, $\left.\mathrm{CDCl}_{3}\right): \delta 162,150,148,147.9,136,129.7,125,120,72.4,21.6$, $21.5 \mathrm{ppm}$. LRMS (+FAB) $\mathrm{m} / z$ calcd for $\mathrm{C}_{19} \mathrm{H}_{17} \mathrm{~N}_{3} \mathrm{O}_{5}(\mathrm{M}+\mathrm{H})^{+}$: 397.09; Found: 397.5.

2-Ethoxy-4,5-bis(3-nitrophenyl)- 6H-1,3-oxazin-6-one (8): White solid; IR $\left(\mathrm{KBr}, v_{\max }, \mathrm{cm}^{-1}\right)$ : 1761, 1614, 1311 and 1091; ${ }^{1} \mathrm{H}$ NMR (500 MHz, $\left.\mathrm{CDCl}_{3}\right): \delta 7.64-7.15$ (m, 8H), 4.2-4.02 $(\mathrm{q}, 2 \mathrm{H}, J=7 \mathrm{~Hz}), 1.27-1.22(\mathrm{t}, 3 \mathrm{H}, J=7 \mathrm{~Hz}) ;{ }^{13} \mathrm{C} \mathrm{NMR}(75.47$ $\left.\mathrm{MHz}, \mathrm{CDCl}_{3}\right): \delta 162.2,133.1,132.8,132.3,132.1,129.8,128.7$, 128.6, 123.3, 61.2, 61.1, 14.7 ppm. LRMS (+FAB) $\mathrm{m} / \mathrm{z}$ calcd for $\mathrm{C}_{18} \mathrm{H}_{13} \mathrm{~N}_{3} \mathrm{O}_{5}(\mathrm{M}+\mathrm{H})^{+}$: 383.08; Found: 383.51 .

2-(Isopropoxy)-4,5-bis (3-bromophenyl)-6H-1,3oxazin-6-one (9): White solid; IR $\left(\mathrm{KBr}, v_{\max }, \mathrm{cm}^{-1}\right): 1759$ and 1609 ; ${ }^{1} \mathrm{H}$ NMR $\left(300 \mathrm{MHz}, \mathrm{CDCl}_{3}\right): \delta$ 7.92-7.18 (m, 8H), 5.180$5.091(\mathrm{~m}, 1 \mathrm{H}), 1.35(\mathrm{~d}, 6 \mathrm{H}, J=6.5 \mathrm{~Hz}) ;{ }^{13} \mathrm{C}$ NMR $(125 \mathrm{MHz}$, $\left.\mathrm{CDCl}_{3}\right): \delta 162.4,150,139,131,130,125,124.6,120.6,64,23$ ppm. LRMS (+FAB) $m / z$ calcd for $\mathrm{C}_{19} \mathrm{H}_{15} \mathrm{NO}_{3} \mathrm{Br}_{2}(\mathrm{M}+\mathrm{H})^{+}: 462.94$; Found: 462.85.

2-Ethoxy-4,5-bis(3-bromophenyl)- 6H-1,3-oxazin-6one (10): White solid; IR $\left(\mathrm{KBr}, v_{\max }, \mathrm{cm}^{-1}\right): 1759,1609$ and $1611 ;{ }^{1} \mathrm{H}$ NMR (500 MHz, $\mathrm{CDCl}_{3}$ ): $87.8-7.2(\mathrm{~m}, 8 \mathrm{H}), 5.180-5.14$ $(\mathrm{m}, 1 \mathrm{H}, J=6 \mathrm{~Hz}), 1.35(\mathrm{~d}, 6 \mathrm{H}, J=6.5 \mathrm{~Hz}) ;{ }^{13} \mathrm{C} \mathrm{NMR}(75.47 \mathrm{MHz}$, $\left.\mathrm{CDCl}_{3}\right): \delta 162.4,149,139,131,125,123,120,55.16 \mathrm{ppm}$. LRMS (+FAB) $\mathrm{m} / \mathrm{z}$ calcd for $\mathrm{C}_{18} \mathrm{H}_{13} \mathrm{NO}_{3} \mathrm{Br}_{2}(\mathrm{M}+\mathrm{H})^{+}$: 448.93; Found: 448.34 .

\section{RESULTS AND DISCUSSION}

Despite their potential utility, zwitterions are rarely used in synthetic organic chemistry when compared with other reactive intermediates. However, we have employed the utility of Huisgen zwitterion for the synthesis of various oxazinones (1-10).

The mechanism of the reaction is explained by the nucleophilic attack of Huisgen zwitterion on cyclopropenone followed by internal cyclization to yield corresponding oxazinone (Scheme-II).

\section{Conclusion}

We have unravelled a facile and novel route for the synthesis of oxazinones and successfully employed it for a series of diaryl cyclopropenones with various dialkyl azodicarboxylates. It is 


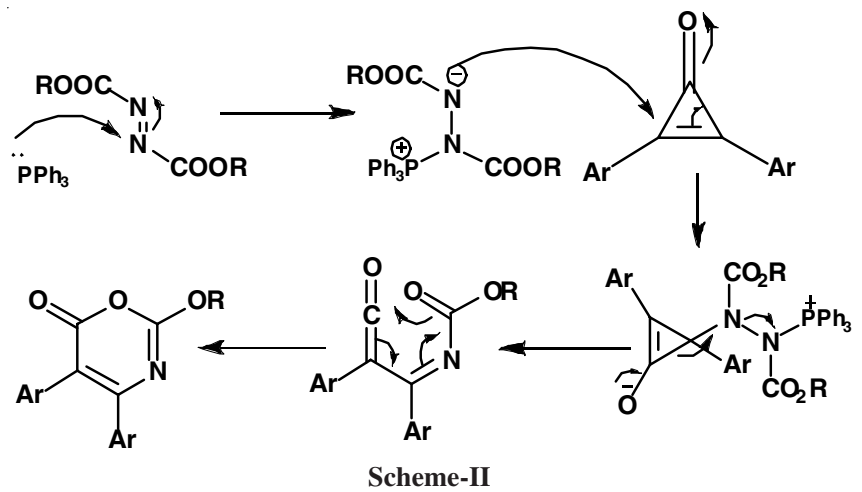

noteworthy that the reactivity of cyclopropenones, the ambident electrophiles, towards Huisgen zwitterions is explored for the first time. It may also be mentioned that oxazinones are important compounds since many of them are reported to posess antimicrobial and antifungal activities [13,14].

\section{REFERENCES}

1. V. Nair, C. Rajesh, A.U. Vinod, S. Bindu, A.R. Sreekanth, J.S. Mathen and L. Balagopal, Acc. Chem. Res., 36, 899 (2003); https://doi.org/10.1021/ar020258p.

2. V. Nair, R.S. Menon, A.R. Sreekanth, N. Abhilash and A.T. Biju, Acc. Chem. Res., 39, 520 (2006); https://doi.org/10.1021/ar0502026.
3. Y.-L. Shi, and M. Shi, Org. Lett., 7, 3057 (2005); https://doi.org/10.1021/ol0510441.

4. D.W. Allen, Organophosphorus Chem., 44, 1 (2015); https://doi.org10.1039/9781782622765-00001.

5. R. Huisgen, E. Brunn, R. Gilardi and I. Karle, J. Am. Chem. Soc., 91, 7766 (1969); https://doi.org/10.1021/ja50001a054.

6. P. Narender, U. Srinivas, M. Ravinder, B.A. Rao, K. Harakishore, C. Ramesh, B.S. Gangadasu, N. Murthy and V.J. Rao, Biorg. Med. Chem., 14, 4600 (2006); https://doi.org/10.1016/i.bmc.2006.02.020.

7. M. Girard, P. Murphy and N. Tsou, Tetrahedron Lett., 46, 2449 (2005); https://doi.org/10.1016/j.tetlet.2005.02.051.

8. M. Marigo, K. Juhl and K.A. Jørgensen, Angew. Chem. Int. Ed., 42, 1367 (2003); https://doi.org/10.1002/anie.200390350.

9. R. Breslow, T. Eicher, A. Krebs, R.A. Peterson and J. Posner, J. Am. Chem. Soc., 87, 1323 (1965);

https://doi.org/10.1021/ja01084a029.

10. C.W. Bird and A.F. Hamer, Org. Prepn. Proc., 2, 79 (1970); https://doi.org/10.1080/00304947009458607.

11. S.W. Tobey and R. West, J. Am. Chem. Soc., 86, 4215 (1964); https://doi.org/10.1021/ja01073a075.

12. J.E. Marino and A. Resendiz, Org. Lett., 7, 371 (2005); https://doi.org/10.1021/o10480527.

13. S. Saaby, M.A. Bella and K.A. Jørgensen, J. Am. Chem. Soc., 126, 8120 (2004); https://doi.org/10.1021/ja047704j.

14. A.S. Hossan, H.M. Abu-Melha and M.A. Al-Omar, Molecules, 17, 11 (2012); https://doi.org/10.3390/molecules171113642. 